Review

\title{
Potential Health Functions of Collagen Bioactive Peptides: A Review
}

\author{
Mengqian Chen, Yanan Li and "Guangrong Huang \\ Key Lab of Marine Food Quality and Hazard Controlling Technology of Zhejiang Province, \\ College of Life Sciences, China Jiliang University, Hangzhou310018, China
}

\author{
Article history \\ Received: 27-07-2020 \\ Revised: 21-09-2020 \\ Accepted: 24-10-2020 \\ Corresponding Author: \\ Guangrong Huang \\ Key Lab of Marine Food \\ Quality and Hazard Controlling \\ Technology of Zhejiang \\ Province, College of Life \\ Sciences, China Jiliang \\ University, Hangzhou310018, \\ China \\ Email: grhuang@126.com
}

\begin{abstract}
Collagen is the most abundant protein in nature and widely exits in animals. However collagen usage is not good enough, which is a waste of natural resources and an environmental burden. Collagen peptides have some special characters and functions. These functions can decide their usage. Therefore producing, discovering and using new functional collagen peptides are promising way to deal with this issue. Collagen peptides are produced by hydrolysing collagen or gelatin via chemical or biochemical methods. Biochemical hydrolysation is more proper to produce functional collagen peptides than chemical hydrolysation. The collagen hydrolysates or peptides have four levels of functions: Nutrition, basic functions, special biological functions and other useful properties. The nutrition is providing amino acids and small peptides. The basic functions are improving the state of connective tissues, such as skin and bone. The special biology functions include antioxidant, antihypertensive and other bioactive benefits. Other useful functions include antifreeze, metal chelation and forming edible film. All these functions decide that they can be used in food as well as other fields. The present work is a compilation of current information on the preparations and functions of collagen peptides. At last, recommendation for the further research of collagen peptides is proposed.
\end{abstract}

Keywords: Collagen Peptides, Bioactive Peptides, Skin, Antioxidant, Antibacterial

\section{Introduction}

The energy crisis, food crisis and environmental problems have become major global issues. It is imperative to explore new resources and increase the utilization of existing resources. The development and utilization of both biomaterials and bio-energy are methods to resolve these problems. Collagen, which exists in the connective tissue of animals, is the most abundant protein in various vertebrates and invertebrates (Dybka and Walczak, 2009). Almost one-third of mammalian proteins are collagens. Nowadays we should take full use of our resources and reduce the use of unsustainable polluted petrochemical resource. So it is meaningful to develop the usage of collagen.

Collagen is a promising resource as renewable materials, like macromolecule cellulose. It can be used as medical materials, food resources, chemical materials, etc. However the collagen utilization ratio is still very low nowadays, because large quantities of collagen are discarded as waste or underutilized to full advantage in the form of by-products (Pal and Suresh, 2016). Fish bone, skin, scale and animal husbandry contain a large number of collagen and are generally discarded as byproducts (Blanco et al., 2017). In addition, it's not a good protein for human nutrition although the collagen is a very cheap and abundant protein. Collagen's amino acid composition proportion doesn't match human's need and collagen is hard to be digested in gastrointestinal tract and also its bioavailability is low. All of these limit the usage of collagen which is a waste of resources.

Research and development of collagen's new usages, as well as take full use of collagen, are important for us. Collagen can be hydrolyzed to peptides by chemical or biological methods. These collagen peptides may overcome the shortage of collagen including low bioavailability. The direct consumption of collagen peptides would improve collagen's bioavailability greatly. These collagen-rich by-products are often then inedible part of animals. However, collagen peptides can 
be achieved from these inedible portions hydrolyzing collagen. Then collagen peptides are also a promising form of take full use of our collagen sources.

At the same time, collagen derived peptides are an abundant source of functional chemicals. Collagen peptides or hydrolysates have been proven to have lots of special functions, such as benefits for skin and bone (Zdzieblik et al., 2017), antioxidant (Ketnawa et al., 2016), antimicrobial (Ennaas et al., 2016), antifreeze (Cao et al., 2016). These special functions of collagen peptides mean that collagen peptides can be used as functional foods, medicine, cosmetics, food additives and so on. In conclusion, collagen peptides have a bright future in lots of fields.

In this review we focus on the productions and the functions of collagen peptides and also some suggestions about collagen peptides research and development in the future are advised. Hope this article can provide some ideas to the other researchers and engineers about the usage of collagen peptides. This can improve the usage of collagen and stimulate the development of collagen industries.

\section{Preparations of Collagen Peptides}

\section{Collagen and Gelatin}

Collagen is the most abundant organic macromolecules in humans and animals. The collagen is a family of extracellular matrix protein and has at least 28 different types. The collagen contains helical trimeric $\alpha$ chains, which are composed of repeating peptide triplets of glycine-Xaa-Yaa (Xaa and Yaa are often proline and hydroxyproline, respectively). It also has two very short coil fragments at the $\mathrm{N}$ - and $\mathrm{C}$-terminal regions, telopeptides, which are largely made up of lysine and its derivatives (Gómez-Guillén et al., 2011).

Gelatin, which is derived from collagen, is a partial hydrolysate of collagen. The hydrogen and covalent bonds in collagen cleave and the $\alpha$-helix becomes a random coil and then insoluble collagen becomes soluble gelatin (Gómez-Guillén et al., 2011). Gelatin is obtained from heating collagen above the transition temperature of collagen's triple-helix structure (Folk, 2015). In addition, chemicals can also change collagen into gelatin during collagen's extraction.

\section{Hydrolyzation of Collagen and Gelatin}

Low-molecular-weight collagen hydrolysates are generally thought to exert better bioactivities than their larger counterparts (Hong et al., 2019). Therefore, collagen peptides have more important applications after hydrolysis. Like other proteins, collagen and gelatin can also be hydrolyzed via chemicals and enzymes. These methods result in different products with different molecular sizes and amino acid sequences. The characteristics of collagen peptides are decided by the hydrolyzation methods' cleavage sites and action time. In general, chemicals can cleave all the peptide amide bonds. Enzymes are sensitive to some special sites in collagen or gelatin and these sites are decided by the enzyme types.

The products of chemical hydrolysis are often amino acids and small peptides that differ based on the action time, while the products of biochemical hydrolysis are often peptides with different sizes and part of amino acids that also differ based on the types of enzymes and the action time. In Fig. 1, we show the collagen products from different types of hydrolysis.

\section{Chemical Hydrolysis}

A high concentration of both acids and bases can cleave the peptide bonds of collagen. Chemical hydrolysis is cheap and simple to produce collagen hydrolysates or peptides. Acid-alkaline hydrolysis methods have short hydrolysis time and are applicable to industrial processes for they are cost effective and simple operative (Pal and Suresh, 2016). However, acid-alkaline hydrolysis is hard to control and the products are unpredictable. In addition, both acids and bases damage the amino acids.

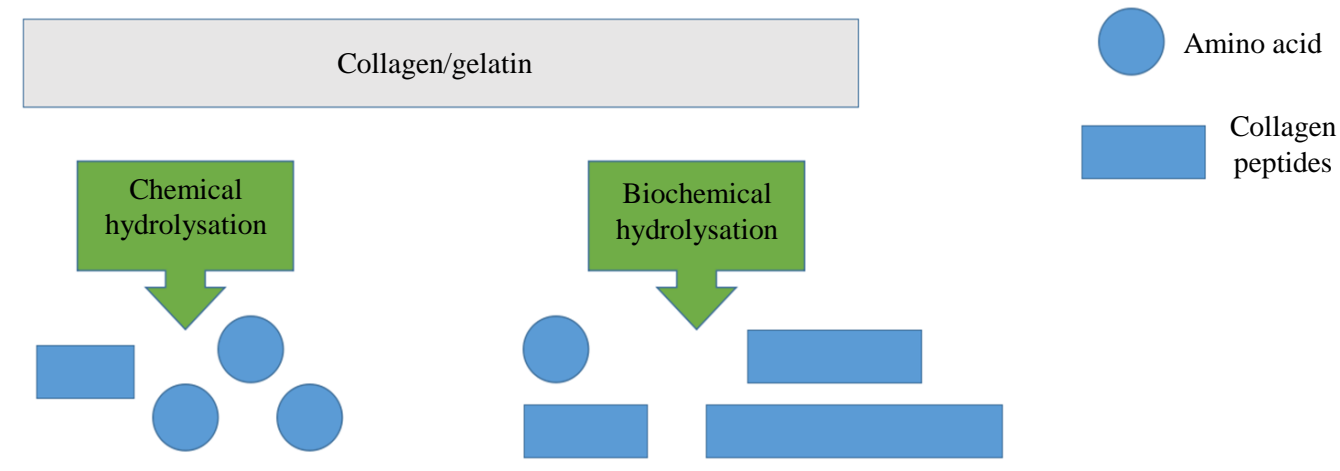

Fig. 1: The products of collagen or gelatin hydrolysates. The chemical hydrolysation of them produces amino acids and small peptides and the products of biochemical hydrolysation are different size of peptides and part of amino acids 
Alkaline conditions can destroy amide nitrogen, transform arginine to ornithine and transform L-amino acids to D-amino acids (Liu et al., 2011). Acids can destroy the $\mathrm{R}$ groups of glutamine, asparagine, tryptophan and others. Then the preparation of collagen peptides via chemical hydrolysis is not very common. What's more, the uses of strong acids in the chemical hydrolysis cause some environment problems (Liu et al., 2011). In addition, collagen is often extracted via the assistance of acid or base. During the extraction, the collagen may be broken by solvent. The acidic collagen extracts acquired from the skin of the silver carp contain considerable quantities of small peptides (Wojtkowiak et al., 2016).

\section{Biochemical Hydrolysis}

Biochemical hydrolysis is another way to produce collagen hydrolysates or peptides. Collagen peptides/hydrolysate can be gained from hydrolysis of collagen by using exogenous and/or endogenous enzymes such as fermentation, gastrointestinal digestion and enzymatic hydrolysis (Pal and Suresh, 2016). Of all these methods, enzymatic hydrolysis is used most commonly. Compared with chemical hydrolysis, enzymolysis is more stable and mild. In addition, the products, e.g., peptides or hydrolysates, can be controlled and predicted.

The hydrolysates of collagen have different amino acid sequences and properties. These differences base on the different enzymes and hydrolysis condition. The enzymes can be non-specific proteinases and/or a specific collagenase or gelatinase. Non-specific proteinases can be trypsin, pancreatin, acidic proteases, neutral proteases, alkaline proteases and a combination of them. Collagenase or gelatinase derived from microorganisms and animals has good specifics and efficiency for collagen, such as the bacterial collagenase obtained from Alcaligenesodorans (Banerjee and Shanthi, 2012). Collagen from pig skin, cattle skin, fish scales and chicken feet are reported hydrolysing by collagenase (Hatanaka et al., 2014). Compared with animal-derived enzymes, enzymes from microorganisms have several advantages, including a wide variety of available catalytic activities and greater $\mathrm{pH}$ values and temperature stabilities (Ovissipour et al., 2012). Therefore, we often use collagenase or gelatinase that come from microorganisms.

Recently, a new green technology called Subcritical Water Hydrolysis (SWH) was used for production of peptides from diverse proteins, including collagen (Ahmed and Chun, 2018). SWH may become one of the most promising methods for producing bioactive peptides from protein; because it only uses water instead of any hazardous solvent and also the hydrolysis processing is very fast, cost-effective and highlyefficient. However, the process needs high pressure and high temperature, which may be one of the weaknesses.

\section{Collagen Hydrolysates and Collagen Peptides}

Collagen is hydrolyzed by different methods to obtain peptides with bioactivities or with smaller sizes. Collagen hydrolysates have been studied as compounds of peptides and amino acids. Collagen hydrolysates are hard to be described and qualified. The entire collagen hydrolysate varies for different species, collagen types and the used enzyme types. Then they are often defined and described by the factors, for example: The hydrolysates of giant catfish skin using porcine trypsin (Ketnawa et al., 2016), hydrolysates of cobia gelatin using pancreatin (Yang et al., 2008) and hydrolysates of thornback ray skin using an enzyme preparation from Bacillus subtilis A26 (Lassoued et al., 2015a). Some collagen hydrolysates will be separated crudely. The separation methods include molecular size (Guillén et al., 2010), mental chelate ability (Guo et al., 2015), graded salting out, ion-exchange chromatography and hydrophobic chromatography.

Among the various collagen hydrolysates, some collagen peptides have been identified and studied. These collagen peptides have been studied carefully and precisely using synthetic peptides. Those collagen peptides were reported to have special functions, such as benefits for skin and bone, antioxidant activity, ACEinhibitory activity and other functions. GHK (glycyl-Lhistidyl-L-lysine, the tri-peptide from osteonectin) is one of the mostly studied collagen peptides. GHK and its copper complex can accelerate wound healing and skin repairing, remodel tissue, etc. (Fu et al., 2015). The collagen tri-peptide fragment GER and its multiple repeats affect the adhesion and spreading of mouse embryonic fibroblasts to different substrates (Ivanova et al., 2012). In addition, a lot of other collagen peptides have also been reported or separated from the collagen hydrolysates, such as DGAR (Sila and Bougatef, 2016), GAOGLOGP (Saiga et al., 2008) and GFOGP (Ichimura et al., 2009). We classified these collagen peptides by their functions in Table 1.

\section{Functions of Collagen Peptides}

Protein's functional and nutritional properties can be improved by hydrolysis (Pal and Suresh, 2016). Peptides from collagen also have various functions. First, as a type of protein fragment, collagen peptides can be digested and absorbed by creatures and used to provide materials and energy for our bodies. Collagen peptides are also fragments of a special protein, collagen, which is the basic of the connective tissue. Then collagen peptides will contribute to collagen growth and the structure of relative tissue. Some collagen peptides with special structures may have special functions, such as antioxidation and antihypertensive activities. Beside these bio-functions, collagen peptides also have other properties that can be 
used, e.g., antifreeze and antimicrobial activities and used as an emulsifier and de-foaming agent. In the Fig. 2, we show the functions of collagen peptides.

\section{Nutrition}

Collagens are the major fibrous component of the animal extracellular matrix and the most abundant protein in nature. As one of biological resources, collagen is regeneration and abundant. These collagens are a promising resource for foods just like the grain, so do the peptides derived from collagens. What's more collagen peptides are safe and low allergenicity. Some collagen peptides have been designated as Generally Recognized As Safe (GRAS) food products or food additives in USA (Dybka and Walczak, 2009). Then collagen peptides are potential and functional food resources.

Table 1: The collagen peptides with special biological functions or properties

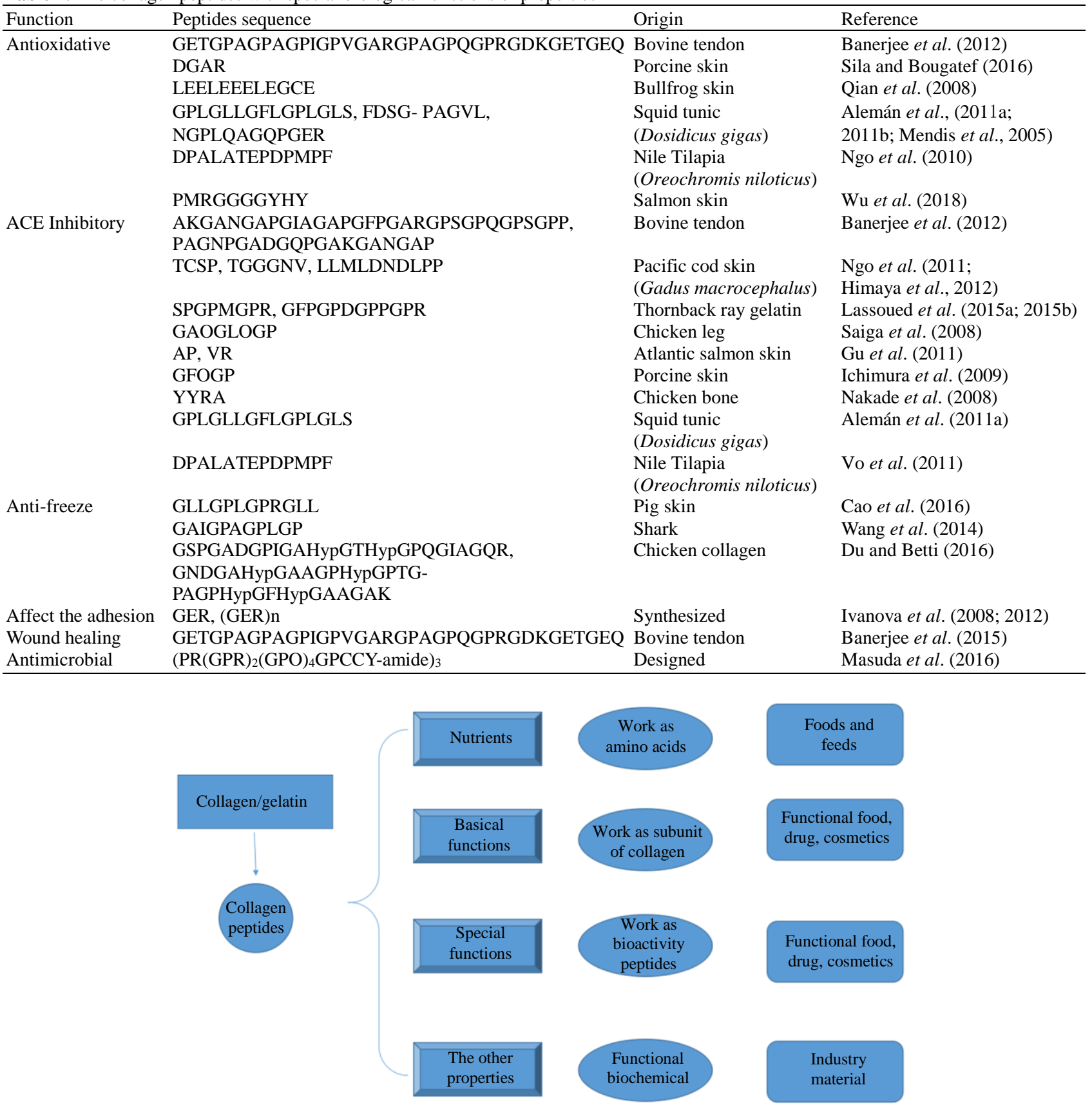

Fig. 2: The function of collagen peptides. The peptides derived from collagen have four levels of functions and all of these functions have their usage 
Collagen and its hydrolysates do not have the proper amino acid composition for human because they do not contain sufficient amounts of $Y, W$ and $F$ residues. However, they still contain almost all of the natural amino acids and are rich in glycine, praline and so forth. Collagen peptides or hydrolysates provide amino acids for people with anorexia, anaemia and for vegetarians whose diet is lack of meat (Dybka and Walczak, 2009) and collagen peptides can be used in combination with other amino acids, such as tryptophan to overcome its low content of some essential amino acids. Due to presented, collagen peptides are still a good source of our foods.

Additionally, collagen peptides have a smaller size than collagen and this can help overcome the shortfalls of collagen, e.g., difficult digestion and low bioavailability. Collagen peptides can be digested into small molecules more easily than collagen. What's more, some collagen peptides, such as di-peptides and tripeptides, can be directly absorbed (Freeman, 2015). Collagenase hydrolyzes collagen or gelatin into tripeptides start with the G, such as GPHyp, GPA and GPHyp. Collagen tri-peptides are absorbed more efficiently than other collagen peptides in humans (Yamamoto et al., 2016). Then collagen peptides are better foods or foods additives than collagen.

\section{Basic Functions}

Collagen is a major material in connective tissues, e.g., skin, bone, cartilage and so on. Permanent collagens exchange processes in our body takes place during the whole human life and old fibrils are replaced by new one all the time (Dybka and Walczak, 2009). Collagen peptides, a subunit of collagen, will contribute to the formation of collagen and the statue of connective tissues. Then the properties of connective tissue would be affected by collagen peptides intake. What's more, the collagen hydrolysates/peptides can perfect the symptoms of some connective tissues diseases or disorders. Skin and cartilage are two categories containing lots of collagen and the benefit effects of collagen peptides are studied most. Besides, tendons, blood vessels, intestines also contain large quantities of collagen. Then collagen peptides may also contribute to these organs' functions or situation. For example, collagen peptides can increase blood vessel's elasticity and be used for high blood pressure adjuvant therapy. Also, the collagen peptides administration prevents intestinal barrier disruption following burn injury (Chen et al., 2019).

\section{Skin Benefits}

Skin contains large quantities of collagen. Collagen and its hydrolysates can be used to construct skin and improve skin's properties. Then oral administration and external application of collagen peptides would benefit the skin and improve the skin's properties. Daily oral supplementation with collagen peptides improves skin hydration, elasticity and slows down skin aging and has a protective effect on joint health (Choi et al., 2014; Pyun et al., 2012; Czajka et al., 2018).

A collagen tri-peptide significantly improved both the moisture levels and skin colour (Berardesca et al., 2009). Collagen peptides can also help our skin cape with some bad situation. Ingestion of collagen peptides improves the skin properties of women during winter (Matsumoto, 2006). The skin benefit effects are also found in the skin of pigs (Matsuda et al., 2006), mice (Pyun et al., 2012; Okawa et al., 2012) and others, with the phenomenon of increased fibroblast density. Zague et al. (2018) found collagen hydrolysates treatment notably modulated cell metabolism and increased the content of procollagen I in monolayer model and the human dermal equivalent model.

At the same time collagen bioactive peptides can also protect skin from damage and heal all kinds of damage. These damages include UVB radiation, photothermolysis treatments, dry skin, sensitive atopic skin and cutaneous wounds. Collagen peptides from bovine tendon, chum salmon (Oncorhynchusketa) and jellyfish accelerate the wound healing process in rats (Wang et al., 2015a; Banerjee et al., 2015; Choi et al., 2014; Felician et al., 2019). Rapid wound closure activity of collagen peptides from a bovine Achilles tendon are also reported in women (Banerjee et al., 2014). Collagen peptides are also good to all kinds of slight injury. Collagen peptides treatment appears to be an effective and conservative therapy for UVB radiation (Pyun et al., 2012), photothermolysis treatment (Choi et al., 2014) and UVirradiation (Zhang et al., 2017) by decreasing the abnormal elastic fibre formation, suppressing epidermal hyperplasia, increasing skin hydration and soluble type-I collagen content, etc. Besides, oral administration of collagen peptides also can improves dry skin. Okawa et al. (2012) found that collagen peptides normalize axonguidance factors in the epidermis and reduce pruritus in dry skin model mice induced by acetone.

\section{Bone and Cartilage Benefits}

Collagens are also the major material in bone and cartilage. Similarly, collagens and their hydrolysates can also benefit the bone and cartilage. Osteoporosis and osteoarthritis are two kinds of disorders or diseases relative to the bone and cartilage and their therapeutic affect nowadays are limited in many patients and there is not a perfect treatment for these two disorders (Alemán and Martinezalvarez, 2013). New and safer compounds have been used in treatment for they can repair the damaged articular cartilage or at least decelerate its progressive degradation (Kanshana et al., 2018). Beside the glucosamine, methyl-sulfonylmethane, chondroitin sulphate, collagen hydrolysates are 
also considered as symptomatic pharmaceutical drugs (Alemán and Martinezalvarez, 2013).

Osteoporosis has lots of symptoms: Pain, bone fragility and easy to fracture for loss of calcium and other mineral elements. All these symptoms would affect our life. Collagen peptides have been reported that it can prevent osteoporosis or treat it. Collagen peptides extracted from fish scales prevent osteoporosis and assist in bone remodelling (Hu et al., 2016). Collagen peptides from deer sinew prevent bone loss in ovariectomized rats (Zhang et al., 2014). The intaking of collagen peptides increases bone mineral density and bone markers in postmenopausal women (König et al., 2018). Collagen peptides apparently stimulate the calcification of human osteoblastic cells in cultures and increase the production of type I collagen (Tsuruoka et al., 2007). The bovine bone collagen peptides may prevent agerelated bone loss and improve bone microarchitecture by increasing collagen synthesis and inhibiting bone absorption (Song et al., 2019).

Osteoarthritis is a joint disease which involves the joint cartilage and its associated structures (Alemán and Martinezalvarez, 2013). A lot of literatures have reported that collagen peptides or hydrolysates can be benefit for some diseases. Collagen peptides isolated from pork skin and bovine bone are effective supplements to improve physical problems associated with osteoarthritis (Kumar et al., 2015). The supplementation of collagen peptides in young adults with functional knee problems led to an improvement in activity-related joint pain (Zdzieblik et al., 2017). Collagen peptides have the potential to exert a chondroprotective action on osteoarthritis by inhibiting matrix metalloproteinase-13 expression and type II collagen degeneration (Isaka et al., 2017). Periodic injections of collagen peptides delay cartilage degeneration in rabbit experimental osteoarthritis by promoting the synthesis of type II collagen and prevent proteoglycan loss in rabbits (Naraoka et al., 2013).

Collagen peptides or hydrolysates can also regular gene expression related to osteogenetic differentiation (Wang et al., 2015b), promoting bone healing process (Hata et al., 2008). In conclusion collagen hydrolysates are promising therapeutic agent in the treatment of bone and cartilage disorders, such as: Osteoarthritis and osteoporosis (Wu et al., 2017).

\section{Special Bio-Functions}

Structure decides the function and collagen peptides with a variety of structures have a variety of functions. Some collagen hydrolysates or peptides are reported to have special bioactivities, such as antioxidant, antihypertensive and anti-cancer activities. These peptides are called cryptic peptides or cryptides (Banerjee and Shanthi, 2016). Collagen bioactive peptides are mainly obtained by enzymatic hydrolysis and are more popular for their safety.

\section{Antioxidant}

Many diseases and disorders, such as cancer, mutations, age, diabetes, cardiovascular disease, Alzheimer's, are related to the fail of antioxidant system in our body. Antioxidants can deal with this problem and improve life expectancy. The antioxidant properties of peptides have been intensely studied for food proteins can inhibit lipid peroxidation (Banerjee and Shanthi, 2016). Lots of collagen peptides or hydrolysates have been proved having antioxidant activities. Peptides from enzyme hydrolysates of discarded fish processing wastes exhibit antioxidant property (Sila and Bougatef, 2016). Collagen hydrolysates in snakehead murrel (Channastriata) skins demonstrate significant antioxidant activities compared with that of butylated hydroxytoluene, vitamin C and E (Sinaga, 2015). Collagen peptides from three types of abalone foot muscles have antioxidant activities in vitro and in vivo (Guo et al., 2016). One bioactive peptide isolated from a bovine tendon collagen confers protection to cells against oxidative stress (Banerjee et al., 2014). The royal jelly-collagen peptide has also ability of protection against oxidative stress and anti-aging effect (Qiu et al., 2020). Collagen peptides are promising antioxidant which deserves further study.

\section{ACE Inhibitory Activity}

Angiotensin I-Converting Enzyme (ACE) inhibitory agents can inhibit the catalyze degradation of bradykinin. However ACE inhibitor used now have some side effects. Then ACE inhibitor form protein will be very promising for their safety. Some collagen peptides have been reported have anti-hypertensive and ACE-inhibitory activities. These ACE inhibitory peptides are thought containing 10-12 amino acids and having a hydrophobic $\mathrm{C}$ terminal. Collagen peptides from milk fish (Chanoschanos) skin possess ACE inhibitor activities (Baehaki et al., 2016). Two peptides from bovine collagen exert anti-hypertensive effects in vivo (Yu et al., 2016). ACE inhibitory peptides GP and GFHypGP, isolated from porcine skin collagen hydrolysates, also had an antihypertensive effect on spontaneously hypertensive rats (Masuda et al., 2018). Peptides from pig femoral bones have anti-hypertensive and ACE inhibitory activities (Liu et al., 2014).

Besides anti-hypertensive and ACE-inhibitory activities, some collagen peptides are also reported to have anti-atherosclerosis and anti-cholesterol activities. Peptides from turkey head collagen can bind cholic and deoxycholic acids and significantly inhibit plasma amine oxidase in a dose- and time-dependent manner (Khiari et al., 2014). Collagen peptides inhibit atherosclerosis development (Tang et al., 2015). Some collagen hydrolysates from snakehead murrel 
(Channastriata) skin demonstrate anti-cholesterol activities comparable to that of pravastatin (Sinaga, 2015). Hypertension, atherosclerosis and cholesterol are three problems related to cardiovascular and cerebrovascular diseases. Peptides from collagen have been proven to have functions to release or cure these problems.

\section{Antimicrobial Activity}

Although there are lots of potent antimicrobial compounds nowadays, antimicrobial peptides are safer in the food safety and food protection as natural products. Antimicrobial peptides have a broad activity spectrum and a rapid microbe killed effect (Alemán and Martinezalvarez, 2013). There have some reports that collagen peptides or hydrolysates have antimicrobial activity (Gómez-Guillén et al., 2011). A designed peptide based on collagen-like (G-P-Yaa)-repeating sequences showed activity against gram-negative Escherichia coli and gram-positive Bacillus subtilis (Masuda et al., 2016). Collagen peptide derived from fish gelatin has antimicrobial activity because it contains hydrophobic motif of GPA triplets in structure (Zhang et al., 2017). Peptide fractions from tuna and squid skin gelatin within a range of $1-10 \mathrm{kDa}$ and $<1 \mathrm{kDa}$ are reported having antimicrobial activity (Guillén et al., 2010).

\section{Other Biofunctions}

In addition to the two functions above, collagen peptides also have other biological functions. These biological functions include anti-cancer activity, opioidlike activity, etc.

Collagen-peptide ingestion suppresses allergic responses by skewing the balance of CD4+ $\mathrm{T}$ cells toward Th1 and Treg cells (Venkatesan et al., 2017). Collagen peptides demonstrated a comparable effect with glutamine in ameliorating post-burn inflammatory responses in mice with burns and the peptides could be considered as a potential immunonutritional supplement in external nutrition to improve post-burn outcomes in burn patients (Chen et al., 2017a).

In addition, collagen peptides are reported to have satiety, opioid-like activity, calciotropic activity (CGRPlike molecules) (Alemán and Martinezalvarez, 2013). Low molecular weight collagen peptides have strong activities for protecting the tight junction barrier function against TNF- $\alpha$ stimulation in Caco-2 cells (Chen et al., 2017b). Collagen peptides from milk fish (Chanoschanos) skin possess anti-cancer activity (Baehaki et al., 2016). Collagen peptides enhanced hyaluronic acid production in human dermal fibroblasts in vitro and in murine skin in vivo (Okawa et al., 2012).

\section{Useful Properties}

In addition to these bio-functions, collagen peptides also have various other properties. The properties of collagen peptides will contribute to their usage. Collagen peptides have been reported to have antifreeze activity, metal ion chelate abilities, film-form and promoting gel hardness.

\section{Antifreeze}

Antifreeze activity is a popular issue in industry. Antifreeze agent can preserve the structure, texture and quality of frozen foods. For example, antifreeze agent can inhibit the form the crystal and keep the texture delicate. Specific collagen peptides have been reported can inhibit ice crystal growth. Collagen peptides from pig skin have cryoprotective effects on conventional dough products (Chen et al., 2016). Collagen peptides from chicken inhibit ice crystal growth in sucrose model systems (Du and Betti, 2016). Collagen peptides from porcine greatly elevate the glass transition temperature of ice cream by binding with ice (Cao et al., 2016).

Beside the potential application in food preservation, collagen peptides with antifreeze and cryoprotection activity also take effect on cryo-protection of cells and tissues (Nguyen et al., 2018). Collagen peptides from pig skin have antifreeze effects on freeze-dried Streptococcus thermophiles, because they interact with cell membrane phospholipids during freeze drying (Wang et al., 2015c). A peptide derived from shark skin collagen hydrolysates also has hypothermia protective activity on Lactobacillus bulgaricus (Wang et al., 2014). The collagen hydrolysates can promise alternative antifreeze proteins. The most studied ones, fall into two groups: Antifreeze Proteins (AFP) and Antifreeze Glycoproteins (AFGP).

In addition to inhibiting ice nucleation, these proteins inhibit icecrystal growth and change the crystal habit of ice by binding to small ice crystals. One of the common features among these antifreeze proteins is that their ice binding face is flat and the distance between oxygen atoms on this face is about the same as that in ice nuclei, that is, about $4.52 \AA$ (Kumar et al., 2015). Molecular modeling of model gelatin peptides revealed that they form an oxygen triad plane at the $\mathrm{C}$ terminus with oxygen-oxygen distances similar to those found in ice nuclei. Binding of this oxygen triad plane to the prism face of ice nuclei via hydrogen bonding appears to be the mechanism by which gelatin hydrolysate might be inhibiting ice crystal growth in ice cream mix (Damodaran, 2007).

In nature, antifreeze proteins exist in a wide range of living organisms, such as Antarctic fish, insects, plants, bacteria and fungi, all thriving in a sub-zero environment. These antifreeze substances are synthesized in their bodies and are believed to play a role in protecting biological tissues from freezing damage during harsh winters or in cold surroundings, through noncolligatively depressing the freezing point without affecting the melting point (thermal hysteresis proteins) 
and/or inhibiting ice crystal growth (ice-structuring proteins) in their body fluids.

\section{Mental Ion Chelate Ability}

Chelateable peptides protect minerals from the inhibition effect of phytic acid or other inhibitors in the gastrointestinal tract ( $\mathrm{Li}$ et al., 2017). Therefore, the mineral will have a higher bioavailability with the exit of chelateable collagen peptides. This property of collagen peptides can be used to promote mineral absorption for the mineral deficiencies would affect people's health. Some collagen peptides have been reported as mineral absorption promoters. Collagen peptide chelated mineral $\left(\mathrm{Ca}^{2+}\right.$, Iron, $\left.\mathrm{Zn}^{2+}, \mathrm{Mg}^{2+}\right)$ products can be used in foods, feeds and pharmaceuticals (Wu et al., 2019). Several peptides from Alaska Pollock skin collagen have a mineral $\left(\mathrm{Ca}^{2+}, \mathrm{Fe}\right.$ and $\left.\mathrm{Cu}^{2+}\right)$ chelation ability. In addition, they have the potential to be used as functional ingredients to manage mineral deficiencies (Guo et al., 2015).

Calcium is an element that studied most often. Tilapia scale collagen hydrolysates can chelate with $\mathrm{Ca}^{2+}$ (Liao et al., 2020). In addition, these hydrolysates are potential functional foods with a calcium-supplement effect. Collagen peptides from deer sinew significantly increase the levels of serum calcium (Zhang et al., 2014). Besides, collagen peptides can also chelate with iron (Guo et al., 2013), copper (Li et al., 2015) and promote their absorption.

\section{Other Properties}

In addition, collagen peptides have lots of other properties. These properties determine their other usages, e.g., surfactant, gel-form and film-form. As a zwitter-ion, collagen peptides act as a surfactant. They can act as an emulsifier, foaming agent, de-foaming agent, etc. The gel-form and film-form are properties of the collagen or gelatin. Collagen peptides can also contribute to forming a gel or film. Some big collagen peptides even have the properties of gels or films. In addition, adding a certain concentration of collagen peptides can promote the hardness of agar gel (Yoshimura et al., 2007).

\section{Recommendation for Further Research}

The remarkable growth of bioactive peptide development in the past decade has led to a large number of market approvals and the market value is expected to hit $\$ 25$ billion by 2018 (Daliri et al., 2018). As food derived bioactive peptides, collagen peptides have huge potential in functional foods, cosmetic and medicine industry. Collagen peptides will be interested for more and more researchers, manufacturer and consumers.

The applications of collagen peptides should be promoted. The benefits of collagen peptides will contribute to their development. If the commercialization of collagen peptides is successful, its development will be ensured. In addition, people will be motivated to research and develop the applications. We should also encourage researchers to develop new production methods for collagen peptides. Those products should be used to replace the production of petrochemical materials to help our fragile environment.

We should develop collagen peptides from marine environments. Marine ecosystems are huge reservoirs of collagen. However, most marine collagen is discarded or used as low value feed. Additionally, marine collagen is easy to denature, especially collagen that derived from deep sea or cold zone animals (Gómez-Guillén et al., 2011). Marine collagen can be used in gelatin form and marine gelatin (especially gelatin from cold water) has a weaker gel strength and lower gelling and melting temperatures than that of non-marine gelatine (Gómez-Guillén et al., 2011). These drawbacks limit their usage as gel-forming materials. Marine collagen can be used in its hydrolysis form to overcome the drawback that it is easily denatured and its usage will relieve the shortage of terrestrial collagen. Terrestrial collagen cannot meet the requirements for development due to the population, epidemic diseases in livestock and religion reasons.

The structure-activity relationship is the key point for collagen peptide use as an active ingredient in food. Structure determines the function. We should determine which structure characteristic determines a function and which factor affects the function. These rules will allow us to find or synthesis more effective collagen peptides. In addition, the mechanisms of collagen peptide functions are also important.

Try to get help from other disciplines, especially informatics. With the assistant of bioinformatics, finding functional collagen peptides is easy and we can predict that which food produce a special peptides during its digest. Besides we also need the assistant of bioengineering. We can produce a large number of target collagen peptide by fermentation of the engineering bacteria.

The toxicology of collagen peptides should be noted because they are used in foods, drugs and cosmetics. Although collagen and its hydrolysates are relatively safe and minimally allergenic, it is possible that some of them have a strong allergen city and some protein hydrolysates may contain prions. The collagen hydrolysates from cattle with mad cow disease may contain some prions.

\section{Conclusion}

Collagen or gelatin is a plentiful biomaterial. Collagens, gelatins and their hydrolysates can be used as foods, foods additives, functional foods, medicine and cosmetics. However, they are often used as low value materials or discarded. The full use of collagen or gelatin can be realized via hydrolysis methods.

Collagen or gelatin can be hydrolysed using different types of enzymes or chemicals to produce collagen peptides. Chemical hydrolysis is cheap but difficult to 
control. It often produces amino acids and small peptide fragments. In contrast, biochemical hydrolysis is predictable and stable. Hydrolysis with enzymes often results in different sizes of peptides that we can predict.

These hydrolysates or collagen peptides have a lot of functions. Collagen peptides can act as a provider of amino acids, a collagen component, a bioactive substance, or a functional material. These functions determine that they can be used in functional foods, cosmetic and medicine. In addition to these biofunctions, they can also be used as food additives for their antifreeze and antimicrobial activities or as a surfactant because of their special structures.

\section{Acknowledgement}

This research is supported by the Science and Technology Project of Zhejiang, Province, China [grant number LGN19C200018]. The founding sponsor had no role in the design of the study; in the collection, analyses, or interpretation of data; in the writing of the manuscript and in the decision to publish the results.

\section{Author's Contributions}

Mengqian Chen and Yanan Li: Wrote the paper.

Gangrong Huang: Provides ideas and helped to revise the article.

\section{Ethics}

The authors declare their responsibility for any ethical issues that may arise after the publication of this manuscript.

\section{Conflict of Interest}

The authors declare that they have no competing interests. The corresponding author affirms that all of the authors have read and approved the manuscript.

\section{References}

Ahmed, R., \& Chun, B. S. (2018). Subcritical water hydrolysis for the production of bioactive peptides from tuna skin collagen. The Journal of Supercritical Fluids, 141, 88-96.

Alemán, A., Giménez, B., Montero, P., \& GómezGuillén, M. C. (2011a). Antioxidant activity of several marine skin gelatins. LWT-Food Science and Technology, 44(2), 407-413.

Alemán, A., Giménez, B., Pérez-Santin, E., GómezGuillén, M. C., \& Montero, P. (2011b). Contribution of Leu and Hyp residues to antioxidant and ACEinhibitory activities of peptide sequences isolated from squid gelatin hydrolysate. Food Chemistry, 125(2), 334-341.
Alemán, A., \& Martínez-Alvarez, O. (2013). Marine collagen as a source of bioactive molecules: A review. The Natural Products Journal, 3(2), 105-114.

Baehaki, A., Suhartono, M. T., Sukarno, Syah, D., \& Setyahadi, S. (2016). Collagen Peptides from Fish Skin with Angiotensin I-Converting Enzyme (ACE) Inhibitor and Cancer Antiproliferative Activity. Research Journal of Pharmaceutical Biological and Chemical Sciences, 7(1), 1994-2000.

Banerjee, P., Mehta, A., \& Shanthi, C. (2014). Investigation into the cyto-protective and wound healing properties of cryptic peptides from bovine Achilles tendon collagen. Chemico-Biological Interactions, 211, 1-10.

Banerjee, P., \& Shanthi, C. (2016). Cryptic peptides from collagen: a critical review. Protein and peptide letters, 23(7), 664-672.

Banerjee, P., \& Shanthi, C. (2012). Isolation of novel bioactive regions from bovine Achilles tendon collagen having angiotensin I-converting enzymeinhibitory properties. Process biochemistry, 47(12), 2335-2346.

Banerjee, P., Suguna, L., \& Shanthi, C. (2015). Wound healing activity of a collagen-derived cryptic peptide. Amino Acids, 47(2), 317-328.

Banerjee, P., Suseela, G., \& Shanthi, C. (2012). Isolation and identification of cryptic bioactive regions in bovine Achilles tendon collagen. The protein journal, 31(5), 374-386.

Berardesca, E., Abril, E., Serio, M., \& Cameli, N. (2009). Effects of topical gluco-oligosaccharide and collagen tripeptide $\mathrm{F}$ in the treatment of sensitive atopic skin. International journal of cosmetic science, 31(4), 271-277.

Blanco, M., Vázquez, J. A., Pérez-Martín, R. I., \& Sotelo, C. G. (2017). Hydrolysates of fish skin collagen: An opportunity for valorizing fish industry byproducts. Marine Drugs, 15(5), 131.

Cao, H., Zhao, Y., Zhu, Y. B., Xu, F., Yu, J. S., \& Yuan, M. (2016). Antifreeze and cryoprotective activities of ice-binding collagen peptides from pig skin. Food chemistry, 194, 1245-1253.

Chen, Q., Gao, X., Zhang, H., Li, B., Yu, G., \& Li, B. (2019). Collagen peptides administration in early enteral nutrition intervention attenuates burninduced intestinal barrier disruption: Effects on tight junction structure. Journal of Functional Foods, 55, 167-174.

Chen, Q., Hou, H., Wang, S., Zhao, X., \& Li, B. (2017a). Effects of early enteral nutrition supplemented with collagen peptides on post-burn inflammatory responses in a mouse model. Food \& function, 8(5), 1933-1941. 
Chen, Q., Chen, O., Martins, I. M., Hou, H., Zhao, X., Blumberg, J. B., \& Li, B. (2017b). Collagen peptides ameliorate intestinal epithelial barrier dysfunction in immunostimulatory Caco-2 cell monolayers via enhancing tight junctions. Food \& function, 8(3), 1144-1151.

Chen, X., Wu, J. H., Li, L., \& Wang, S. Y. (2016). The cryoprotective effects of antifreeze peptides from pigskin collagen on texture properties and water mobility of frozen dough subjected to freeze-thaw cycles. European Food Research and Technology, 243(7), 1149-1156.

Choi, S. Y., Kim, W. G., Ko, E. J., Lee, Y. H., Kim, B. G., Shin, H. J., ... \& Lee, H. J. (2014). Effect of high advanced-collagen tripeptide on wound healing and skin recovery after fractional photothermolysis treatment. Clinical and experimental dermatology, 39(8), 874-880.

Czajka, A., Kania, E. M., Genovese, L., Corbo, A., Merone, G., Luci, C., \& Sibilla, S. (2018). Daily oral supplementation with collagen peptides combined with vitamins and other bioactive compounds improves skin elasticity and has a beneficial effect on joint and general wellbeing. Nutrition Research, 57, 97-108.

Damodaran, S. (2007). Inhibition of ice crystal growth in ice cream mix by gelatin hydrolysate. Journal of Agricultural and Food Chemistry, 55(26), 10918-10923.

Daliri, E. B. M., Lee, B. H., \& Oh, D. H. (2018). Current trends and perspectives of bioactive peptides. Critical reviews in food science and nutrition, 58(13), 2273-2284.

Du, L., \& Betti, M. (2016). Identification and evaluation of cryoprotective peptides from chicken collagen: ice-growth inhibition activity compared to that of type I antifreeze proteins in sucrose model systems. Journal of agricultural and food chemistry, 64(25), 5232-5240.

Dybka, K. A., \& Walczak, P. (2009). Collagen hydrolysates as a new diet supplement.

Ennaas, N., Hammami, R., Gomaa, A., Bédard, F., Biron, É., Subirade, M., ... \& Fliss, I. (2016). Collagencin, an antibacterial peptide from fish collagen: Activity, structure and interaction dynamics with membrane. Biochemical and biophysical research communications, 473(2), 642-647.

Felician, F. F., Yu, R. H., Li, M. Z., Li, C. J., Chen, H. Q., Jiang, Y., ... \& Xu, H. M. (2019). The wound healing potential of collagen peptides derived from the jellyfish Rhopilema esculentum. Chinese Journal of Traumatology, 22(1), 12-20.

Folk, J. E. (2015). Fish gelatin: characteristics, functional properties, applications and future potentials. Food Eng. Reviews. 7(1), 33-44.
Freeman, H. J. (2015). Clinical relevance of intestinal peptide uptake. World Journal of Gastrointestinal Pharmacology and Therapeutics, 6(2), 22.

Fu, S. C., Cheuk, Y. C., Chiu, W. Y. V., Yung, S. H., Rolf, C. G., \& Chan, K. M. (2015). Tripeptide-copper complex GHK-Cu (II) transiently improved healing outcome in a rat model of ACL reconstruction. Journal of Orthopaedic Research, 33(7), 1024-1033.

Guillén, G., López Caballero, M. E., Alemán, A., López de Lacey, A., Giménez, B., \& Montero García, P. (2010). Antioxidant and antimicrobial peptide fractions from squid and tuna skin gelatin.

Gómez-Guillén, M. C., Giménez, B., López-Caballero, M. A., \& Montero, M. P. (2011). Functional and bioactive properties of collagen and gelatin from alternative sources: A review. Food hydrocolloids, 25(8), 1813-1827.

Gu, R. Z., Li, C. Y., Liu, W. Y., Yi, W. X., \& Cai, M. Y. (2011). Angiotensin I-converting enzyme inhibitory activity of low-molecular-weight peptides from Atlantic salmon (Salmo salar L.) skin. Food Research International, 44(5), 1536-1540.

Guo, L., Harnedy, P. A., O’Keeffe, M. B., Zhang, L., Li, B., Hou, H., \& FitzGerald, R. J. (2015). Fractionation and identification of Alaska pollock skin collagen-derived mineral chelating peptides. Food Chemistry, 173, 536-542.

Guo, L., Hou, H., Li, B., Zhang, Z., Wang, S., \& Zhao, X. (2013). Preparation, isolation and identification of iron-chelating peptides derived from Alaska pollock skin. Process Biochemistry, 48(5-6), 988-993.

Guo, Z., Hou, X., Zhang, J., Li, L., \& Zhang, H. (2016). Antioxidant activity of collagen peptides from three kinds of abalone foot muscle. J. Shandong Univ. (Nat. Sci.), 51(11), 66-73.

Hata, S., Hayakawa, T., Okada, H., Hayashi, K., Akimoto, Y., \& Yamamoto, H. (2008). Effect of oral administration of high advanced-collagen tripeptide (HACP) on bone healing process in rat. Journal of Hard Tissue Biology, 17(1), 17-22.

Hatanaka, T., Kawakami, K., \& Uraji, M. (2014). Inhibitory effect of collagen-derived tripeptides on dipeptidylpeptidase-IV activity. Journal of Enzyme Inhibition and Medicinal Chemistry, 29(6), 823-828.

Himaya, S. W. A., Ngo, D. H., Ryu, B., \& Kim, S. K. (2012). An active peptide purified from gastrointestinal enzyme hydrolysate of Pacific cod skin gelatin attenuates angiotensin-1 converting enzyme (ACE) activity and cellular oxidative stress. Food Chemistry, 132(4), 1872-1882.

Hong, H., Fan, H., Chalamaiah, M., \& Wu, J. (2019). Preparation of low-molecular-weight, collagen hydrolysates (peptides): Current progress, challenges and future perspectives. Food chemistry, $301,125222$. 
Hu, C. H., Yao, C. H., Chan, T. M., Huang, T. L., Sen, Y., Huang, C. Y., \& Ho, C. Y. (2016). Effects of different concentrations of collagenous peptide from fish scales on osteoblast proliferation and osteoclast resorption. Chin. J. Physiol, 59, 191-201.

Ichimura, T., Yamanaka, A., Otsuka, T., Yamashita, E., \& Maruyama, S. (2009). Antihypertensive effect of enzymatic hydrolysate of collagen and Gly-Pro in spontaneously hypertensive rats. Bioscience, biotechnology and biochemistry, 0909031633-0909031633.

Isaka, S., Someya, A., Nakamura, S., Naito, K., Nozawa, M., Inoue, N., ... \& Kaneko, K. (2017). Evaluation of the effect of oral administration of collagen peptides on an experimental rat osteoarthritis model. Experimental and therapeutic medicine, 13(6), 2699-2706.

Ivanova, V. P., Kovaleva, Z. V., Anokhina, V. V., \& Krivchenko, A. I. (2012). The effect of the collagen tripeptide fragment (GER) on the adhesion and spreading of fibroblasts depends on the properties of adhesive surface. Tsitologiia, 54(11), 823-830.

Ivanova, V. P., Kovaleva, Z. V., Zabelinskii, S. A., Grinchuk, T. M., \& Krivchenko, A. I. (2008). Role of collagen tripeptide fragment GER on activation of adhesion and modification of fatty acid composition in membrane phospholipids of CHOK1 cells. Cell and Tissue Biology, 2(2), 115-122.

Kanshana, J. S., Rebello, S. C., Pathak, P., Kanuri, B. N., Aggarwal, H., Srivastava, V., ... \& Dikshit, M. (2018). Standardized fraction of Xylocarpus moluccensis fruits improve vascular relaxation and plaque stability in dyslipidemic models of atherosclerosis. Journal of ethnopharmacology, 213, 81-91.

Ketnawa, S., Martínez-Alvarez, O., Benjakul, S., \& Rawdkuen, S. (2016). Gelatin hydrolysates from farmed Giant catfish skin using alkaline proteases and its antioxidative function of simulated gastrointestinal digestion. Food chemistry, 192, 34-42.

Khiari, Z., Ndagijimana, M., \& Betti, M. (2014). Low molecular weight bioactive peptides derived from the enzymatic hydrolysis of collagen after isoelectric solubilization/precipitation process of turkey byproducts. Poultry science, 93(9), 2347-2362.

Nakade, K., Kamishima, R., Inoue, Y., Ahhmed, A., Kawahara, S., Nakayama, T., ... \& Muguruma, M. (2008). Identification of an antihypertensive peptide derived from chicken bone extract. Animal Science Journal, 79(6), 710-715.

König, D., Oesser, S., Scharla, S., Zdzieblik, D., \& Gollhofer, A. (2018). Specific collagen peptides improve bone mineral density and bone markers in postmenopausal women-A randomized controlled study. Nutrients, 10(1), 97.
Kumar, S., Sugihara, F., Suzuki, K., Inoue, N., \& Venkateswarathirukumara, S. (2015). A double-blind, placebo-controlled, randomised, clinical study on the effectiveness of collagen peptide on osteoarthritis. Journal of the Science of Food and Agriculture, 95(4), 702-707.

Lassoued, I., Mora, L., Nasri, R., Aydi, M., Aristoy, M. C., ... \& Nasri, M. (2015a). Characterization, antioxidative and ACE inhibitory properties of hydrolysates obtained from thornback ray (Raja clavata) muscle. Journal of Proteomics, 128, 458-468.

Lassoued, I., Mora, L., Nasri, R., Jridi, F., Aristoy, M. C., \& Nasri, M. (2015b). Characterization and comparative assessment of antioxidant and ACE inhibitory activities of thornback ray gelatin hydrolysates. Journal of Functional Foods, 13, 225-238.

Li, H., Low, Y. S. J., Chong, H. P., Zin, M. T., Lee, C. Y., Li, B., ... \& Kang, L. (2015). Microneedlemediated delivery of copper peptide through skin. Pharmaceutical research, 32(8), 2678-2689.

Li, Y., Jiang, H., \& Huang, G. (2017). Protein hydrolysates as promoters of non-haem iron absorption. Nutrients, 9(6), 609.

Liao, W., Chen, H., Jin, W., Yang, Z., Cao, Y., \& Miao, J. (2020). Three Newly Isolated Calcium-Chelating Peptides from Tilapia Bone Collagen Hydrolysate Enhance Calcium Absorption Activity in Intestinal Caco-2 Cells. Journal of Agricultural and Food Chemistry, 68(7), 2091-2098.

Liu, X. H., Li, C., Fu, G., \& Su, Z. (2014). Separation and Purification of Anti-hypertensive Peptides from Pig Femoral Collagen. Food Science, (6), 12.

Liu, Z., Su, Y., \& Zeng, M. (2011). Amino acid composition and functional properties of giant red sea cucumber (Parastichopus californicus) collagen hydrolysates. Journal of Ocean University of China, 10(1), 80-84.

Masuda, R., Kudo, M., Dazai, Y., Mima, T., \& Koide, T. (2016). Collagen-like antimicrobial peptides. Peptide Science, 106(4), 453-459.

Masuda, Y., Ogura, Y., Inagaki, Y., Yasui, T., \& Aizu, Y. (2018). Analysis of the influence of collagen fibres in the dermis on skin optical reflectance by Monte Carlo simulation in a nine-layered skin model. Skin Research and Technology, 24(2), 248-255.

Matsuda, N., Koyama, Y. I., Hosaka, Y., Ueda, H., Watanabe, T., Araya, T., ... \& Takehana, K. (2006). Effects of ingestion of collagen peptide on collagen fibrils and glycosaminoglycans in the dermis. Journal of nutritional science and vitaminology, 52(3), 211-215.

Matsumoto, H. (2006). Clinical effects of fish type I collagen hydrolysate on skin properties. ITE. Lett. Batter. New Technol. Med., 7, 386-390. 
Mendis, E., Rajapakse, N., Byun, H. G., \& Kim, S. K. (2005). Investigation of jumbo squid (Dosidicus gigas) skin gelatin peptides for their in vitro antioxidant effects. Life sciences, 77(17), 2166-2178.

Naraoka, T., Ishibashi, Y., Tsuda, E., Yamamoto, Y., Kusumi, T., \& Toh, S. (2013). Periodic knee injections of collagen tripeptide delay cartilage degeneration in rabbit experimental osteoarthritis. Arthritis research \& therapy, 15(1), 1-10.

Ngo, D. H., Qian, Z. J., Ryu, B., Park, J. W., \& Kim, S. K. (2010). In vitro antioxidant activity of a peptide isolated from Nile tilapia (Oreochromis niloticus) scale gelatin in free radical-mediated oxidative systems. Journal of Functional Foods, 2(2), 107-117.

Ngo, D. H., Ryu, B., Vo, T. S., Himaya, S. W. A., Wijesekara, I., \& Kim, S. K. (2011). Free radical scavenging and angiotensin-I converting enzyme inhibitory peptides from Pacific cod (Gadus macrocephalus) skin gelatin. International journal of biological macromolecules, 49(5), 1110-1116.

Nguyen, C. T., Yuan, M., Yu, J. S., Ye, T., Cao, H., \& $\mathrm{Xu}, \mathrm{F}$. (2018). Isolation of ice structuring collagen peptide by ice affinity adsorption, its ice-binding mechanism and breadmaking performance in frozen dough. Journal of Food Biochemistry, 42(3), e12506.

Okawa, T., Yamaguchi, Y., Takada, S., Sakai, Y., Numata, N., Nakamura, F., ... \& Aihara, M. (2012). Oral administration of collagen tripeptide improves dryness and pruritus in the acetone-induced dry skin model. Journal of Dermatological Science, 66(2), 136-143.

Ovissipour, M., Safari, R., Motamedzadegan, A., \& Shabanpour, B. (2012). Chemical and biochemical hydrolysis of Persian sturgeon (Acipenser persicus) visceral protein. Food and Bioprocess Technology, 5(2), 460-465.

Pal, G. K., \& Suresh, P. V. (2016). Sustainable valorisation of seafood by-products: Recovery of collagen and development of collagen-based novel functional food ingredients. Innovative food science \& emerging technologies, 37, 201-215.

Pyun, H. B., Kim, M., Park, J., Sakai, Y., Numata, N., Shin, J. Y., ... \& Hwang, J. K. (2012). Effects of collagen tripeptide supplement on photoaging and epidermal skin barrier in UVB-exposed hairless mice. Preventive Nutrition and Food Science, 17(4), 245.

Qian, Z. J., Jung, W. K., \& Kim, S. K. (2008). Free radical scavenging activity of a novel antioxidative peptide purified from hydrolysate of bullfrog skin, Rana catesbeiana Shaw. Bioresource Technology, 99(6), 1690-1698.
Qiu, W., Chen, X., Tian, Y., Wu, D., Du, M., \& Wang, S. (2020). Protection against oxidative stress and anti-aging effect in Drosophila of royal jellycollagen peptide. Food and Chemical Toxicology, $135,110881$.

Saiga, A. I., Iwai, K., Hayakawa, T., Takahata, Y., Kitamura, S., Nishimura, T., \& Morimatsu, F. (2008). Angiotensin I-converting enzyme-inhibitory peptides obtained from chicken collagen hydrolysate. Journal of Agricultural and Food Chemistry, 56(20), 9586-9591.

Sila, A., \& Bougatef, A. (2016). Antioxidant peptides from marine by-products: Isolation, identification and application in food systems. A review. Journal of Functional Foods, 21, 10-26.

Sinaga, W. S. L. (2015). Peptides hydrolysate derived from collagen of snakehead murrel (Channa striata) skin demonstrate antioxidant and anticholesterol activities. Unpublished dissertation in partial fulfillment of the requirements for the degree of Magister Science, Bogor Agricultral University, Bogor, Indonesia.

Song, H., Zhang, S., Zhang, L., \& Li, B. (2019). Ingestion of collagen peptides prevents bone loss and improves bone microarchitecture in chronologically aged mice. Journal of Functional Foods, 52, 1-7.

Tang, L., Sakai, Y., Ueda, Y., \& Katsuda, S. (2015). Effects of oral administration of tripeptides derived from type I collagen (collagen tripeptide) on atherosclerosis development in hypercholesterolemic rabbits. Journal of bioscience and bioengineering, 119(5), 558-563.

Tsuruoka, N., Yamato, R., Sakai, Y., Yoshitake, Y., \& Yonekura, H. (2007). Promotion by collagen tripeptide of type I collagen gene expression in human osteoblastic cells and fracture healing of rat femur. Bioscience, biotechnology and biochemistry, 71(11), 2680-2687.

Venkatesan, J., Anil, S., Kim, S. K., \& Shim, M. S. (2017). Marine fish proteins and peptides for cosmeceuticals: A review. Marine drugs, 15(5), 143.

Vo, T. S., Ngo, D. H., Kim, J. A., Ryu, B., \& Kim, S. K. (2011). An antihypertensive peptide from tilapia gelatin diminishes free radical formation in murine microglial cells. Journal of agricultural and food chemistry, 59(22), 12193-12197.

Wang, J., Xu, M., Liang, R., Zhao, M., Zhang, Z., \& Li, Y. (2015a). Oral administration of marine collagen peptides prepared from chum salmon (Oncorhynchus keta) improves wound healing following cesarean section in rats. Food \& Nutrition Research, 59(1), 26411. 
Wang, P., Jiang, J., Pan, D., Sun, Y., Wu, Z., Cao, J., \& Zeng, X. (2015b). Effects of goose collagen and collagen peptide on osteoporosis. International Journal of Food Properties, 19(10), 2190-2201.

Wang, W., Chen, M., Wu, J., \& Wang, S. (2015c). Hypothermia protection effect of antifreeze peptides from pigskin collagen on freeze-dried Streptococcus thermophiles and its possible action mechanism. LWT-Food Science and Technology, 63(2), 878-885.

Wang, S., Zhao, J., Chen, L., Zhou, Y., \& Wu, J. (2014). Preparation, isolation and hypothermia protection activity of antifreeze peptides from shark skin collagen. LWT-Food Science and Technology, 55(1), 210-217.

Wojtkowiak, D., Frydrychowski, A. F., Hadzik, J., \& Dominiak, M. (2016). Identification of Small Peptides of Acidic Collagen Extracts from Silver Carp Skin and Their Therapeutic Relevance. Adv Clin Exp Med, 25(2), 227-235.

Wu, R., Wu, C., Liu, D., Yang, X., Huang, J., Zhang, J., ... \& He, H. (2018). Antioxidant and anti-freezing peptides from salmon collagen hydrolysate prepared by bacterial extracellular protease. Food chemistry, 248, 346-352.

Wu, W., He, L., Liang, Y., Yue, L., Peng, W., Jin, G., \& Ma, M. (2019). Preparation process optimization of pig bone collagen peptide-calcium chelate using response surface methodology and its structural characterization and stability analysis. Food chemistry, 284, 80-89.

Wu, W., Li, B., Hou, H., Zhang, H., \& Zhao, X. (2017). Isolation and identification of calcium-chelating peptides from Pacific cod skin gelatin and their binding properties with calcium. Food \& function, 8(12), 4441-4448.

Yamamoto, S., Deguchi, K., Onuma, M., Numata, N., \& Sakai, Y. (2016). Absorption and urinary excretion of peptides after collagen tripeptide ingestion in humans. Biological and Pharmaceutical Bulletin, 39(3), 428-434.
Yang, J. I., Ho, H. Y., Chu, Y. J., \& Chow, C. J. (2008). Characteristic and antioxidant activity of retorted gelatin hydrolysates from cobia (Rachycentron canadum) skin. Food chemistry, 110(1), 128-136.

Yoshimura, M., Kuwano, T., \& Nishinari, K. (2007). Effects of collagen-peptide on physical properties of agar gels. J. Cookery Sci. Japan. 40(3), 156-165.

Yu, F., Young, J. F., Rasmussen, M. K., Dalsgaard, T. K., Lametsch, R., Aluko, R. E., \& Therkildsen, M. (2016). Angiotensin I-converting enzymeinhibitory peptides from bovine collagen: insights into inhibitory mechanism and transepithelial transport. Food Research International, 89, 373-381.

Zague, V., do Amaral, J. B., Rezende Teixeira, P., de Oliveira Niero, E. L., Lauand, C., \& Machado-Santelli, G. M. (2018). Collagen peptides modulate the metabolism of extracellular matrix by human dermal fibroblasts derived from sun-protected and sun-exposed body sites. Cell biology international, 42(1), 95-104.

Zdzieblik, D., Oesser, S., Gollhofer, A., \& König, D. (2017). Improvement of activity-related knee joint discomfort following supplementation of specific collagen peptides. Applied Physiology, Nutrition and Metabolism, 42(6), 588-595.

Zhang, H., Dong, Y., Qi, B., Liu, L., Zhou, G., Bai, X., ... \& Zhao, Y. (2014). Preventive effects of collagen peptide from deer sinew on bone loss in ovariectomized rats. Evidence-Based Complementary and Alternative Medicine, 2014.

Zhang, L., Zheng, Y., Cheng, X., Meng, M., Luo, Y., \& Li, B. (2017). The anti-photoaging effect of antioxidant collagen peptides from silver carp (Hypophthalmichthys molitrix) skin is preferable to tea polyphenols and casein peptides. Food \& function, 8(4), 1698-1707. 